\title{
Leukocyte Apoptotic Process
}

National Cancer Institute

\section{Source}

National Cancer Institute. Leukocyte Apoptotic Process. NCI Thesaurus. Code C40716.

Any apoptotic process that is observed in leukocytes. 